\title{
A force method model for dynamic analysis of flat-sag cable structures
}

\author{
Xing Ma and John W. Butterworth* \\ Department of Civil and Environmental Engineering, University of Auckland, Private bag 92019, Auckland 1142, \\ New Zealand
}

Received 21 September 2008

Revised 20 December 2008

\begin{abstract}
A new force method is proposed for analysing the dynamic behaviour of oscillating cables with small sags. The accepted dynamic model of such cables reduces to a partial differential equation (the equation of motion) and an integral equation (the compatibility equation). In the paper, D'Alembert's travelling wave solution is applied to the partial differential equation (PDE). Substituting the solution into the compatibility and boundary conditions, the governing equation is obtained in terms of the dynamic tension increment. This equation has been named the force method dynamic equation (FMDE). In this way the infinite-degree-of-freedom dynamic system is effectively simplified to a system with only one unknown. Explicit solutions for both single-span and multi-span cable systems are derived. The natural frequencies obtained from the FMDE are shown to be identical to those deduced using the conventional displacement method (DM). Nonlinear governing equations are developed by considering the effect of quadratic and cubic displacement terms. Finally, two examples are presented to illustrate the accuracy of the proposed force method for single and multi-span cable systems subjected to harmonic forces.
\end{abstract}

Keywords: Force method model, vibration, cable structure, nonlinear analysis, multi-span cable

\section{Introduction}

Cables and cable suspended structures have a wide range of practical applications in both the civil and electrical engineering industries, where their dynamic behaviour is often of crucial importance. The required dynamic analyses have most frequently been carried out in the time domain and based on the displacement method (DM). The prominent linear theory developed by Irvine [4] describes the in-plane and out-of-plane small amplitude free vibration of a suspended elastic cable with small sag. The finite element method has also been used for forced vibration response analysis [7]. However, for cables with complex dynamic geometry, the method needs a quantity of pre-stressed link elements or curved cable elements. To reduce the number of degrees of freedom, low order linear modes are employed to synthesise the dynamic curve of the cable, based on which, discretised models with 2 to 4 displacement modes (d.o.f) have been applied to dynamic analysis of cable-stayed structures $[1,9,10]$. Unfortunately, in some complex circumstances, the conventional displacement method using a few linearised mode functions may lead to inaccurate response prediction. As a new research approach, Ni, Lou and Ko [8] developed a hybrid pseudo-force/Laplace transform method for transient response of suspended cables.

In practical applications, the force method is an ideal structural analysis tool when dealing with pre-stressed structures. You [12] employed the force method to optimise a guyed mast. However, there is no literature available on the use of the force method in dynamic analysis. In dynamic analysis of shallow cables with sag-span ratios smaller than $1 / 8$, the component of additional dynamic tension may be assumed constant in the chord direction if the

\footnotetext{
*Corresponding author. Tel.: +64 93737599 88154; E-mail: jw.butterworth@auckland.ac.nz.
} 


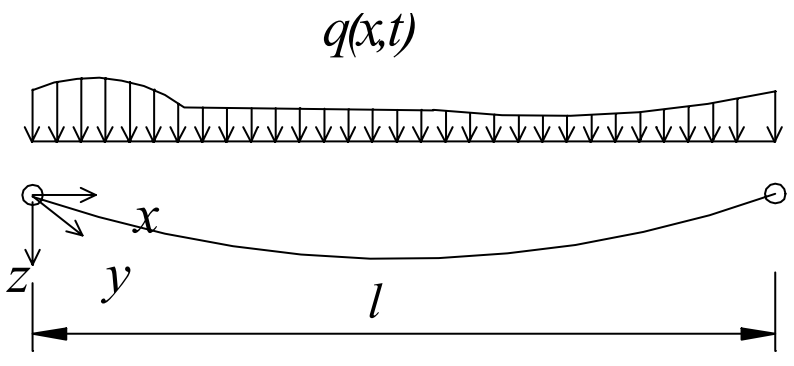

Fig. 1. Model of a suspended cable.

longitudinal inertia forces are omitted $[4,6,9,11,13]$. Thus there is only one unknown in the governing equation if the force method is employed, offering the possibility of reducing the infinite-degree-of-freedom system (deformation method model) to a single-degree-freedom system (force method model) without loss of precision.

For an oscillating shallow cable, the dynamic model reduces to a partial differential equation (equation of motion) and an integral equation (compatibility condition). There are two normal methods for PDE solving [5]: the stationary wave method and the travelling wave method. The stationary wave method is applicable to finite length cables, where a series solution for displacement response can be achieved. This approach is also known as the mode superposition method. However, there is a relatively simple integral formula for displacement response which may be derived based on the travelling wave method. In this paper, in order to employ the travelling wave method to solve the PDE, the support reaction forces are considered as excitations, allowing D'Alembert's solution to be used. Substituting the solution into the compatibility and boundary condition equations leads to the governing equation expressed in terms of dynamic tension - in which form it has been given the name force method dynamic equation (FMDE). Considering the quadratic and cubic terms of the dynamic displacements, nonlinear governing equations are developed. In this way the infinite-degree-of-freedom dynamic system is reduced to a single-degree-of-freedom system.

\section{Linearised undamped model for a single-span cable}

\subsection{Equation of motion and D'Alembert solution}

Consider a transversely loaded single cable spanning a distance $l$, as shown in Fig. 1. For an undamped vertically oscillating shallow cable, the linearised equation of motion is [4].

$$
H \frac{\partial^{2} w}{\partial x^{2}}+h \frac{d^{2} z}{d x^{2}}+q=m \frac{\partial^{2} w}{\partial t^{2}}
$$

where $m$ is the mass density; $H$ and $h(t)$ are respectively the horizontal components of static tension and the increment of dynamic tension; $x$ is the axial coordinate; $w(x, t), q(x, t)$ are respectively the dynamic displacement response and excitation load in the $z$ direction. $z(x)$ is the static equilibrium curve of the cable under static gravity loading $(q=0)$.

Considering support reaction forces as excitations, Eq. (1) is then rewritten as

$$
\frac{\partial^{2} w}{\partial t^{2}}=a^{2} \frac{\partial^{2} w}{\partial x^{2}}+F(x, t)
$$

where

$$
F(x, t)=\left\{\begin{array}{cl}
\frac{h}{m} \frac{d^{2} z}{d x^{2}}+\frac{f_{1} \delta(x)+f_{2} \delta(x-l)+q}{m} & 0 \leqslant x \leqslant l \text { and } t \geqslant 0 \\
0 & \text { others }
\end{array}\right.
$$

and

$$
a=\sqrt{H / m}
$$

If the static equilibrium load includes only the self-weight of the system, we get 


$$
\frac{d^{2} z}{d x^{2}}=-\frac{m g}{H}
$$

$f_{1}(t), f_{2}(t)$ are the support reaction forces and $\delta(x)$ is the unit impulse or Dirac delta function, defined as

$$
\delta(x)=\left\{\begin{array}{c}
\infty x=0 \\
0 x \neq 0
\end{array} \text { and } \int_{-\infty}^{+\infty} \delta(x) d x=1\right.
$$

The D'Alembert solution to Eq. (2) with initial displacement and velocity of zero is

$$
w(x, t)=\frac{1}{2 a} \int_{0}^{t} \int_{x-a(t-\tau)}^{x+a(t-\tau)} F(\xi, \tau) d \xi d \tau
$$

\subsection{Boundary conditions}

The boundary conditions are

$$
w(0, t)=w(l, t)=0
$$

and

$$
\frac{d w(0, t)}{d t}=\frac{d w(l, t)}{d t}=0
$$

Substituting Eq. (7) into Eq. (9) and changing the integral sequence, we get

$$
a \int_{t-l / a}^{t}\left\{q[a(t-\tau), \tau]-\frac{m g}{H} h(\tau)\right\} d \tau+f_{1}(t)+f_{2}\left(t-\frac{l}{a}\right)=0
$$

and

$$
a \int_{t-l / a}^{t}\left\{q[l-a(t-\tau), \tau]-\frac{m g}{H} h(\tau)\right\} d \tau+f_{2}(t)+f_{1}\left(t-\frac{l}{a}\right)=0
$$

where the support forces may be expressed as

$$
\begin{aligned}
& f_{1}(t)=\left\{\begin{array}{cc}
0 & t<0 \\
-f_{2}\left(t-\frac{l}{a}\right)+a \int_{t-l / a}^{t}\left\{\frac{m g}{H} h(\tau)-q[a(t-\tau), \tau]\right\} d \tau & t \geqslant 0
\end{array}\right. \\
& f_{2}(t)=\left\{\begin{array}{cr}
t<0 \\
-f_{1}\left(t-\frac{l}{a}\right)+a \int_{t-l / a}^{t}\left\{\frac{m g}{H} h(\tau)-q[l-a(t-\tau), \tau]\right\} d \tau & t \geqslant 0
\end{array}\right.
\end{aligned}
$$

Differentiating Eq. (7) with respect to $x$ and substituting $x=0$, we get

$$
\frac{\partial w}{\partial x}(0, t)=\frac{1}{2 a m}\left\{\int_{t-l / a}^{t} q[a(t-\tau), \tau] d \tau-\frac{m g}{H} \int_{t-l / a}^{t} h(\tau) d \tau-\frac{1}{a} f_{1}(t)+\frac{1}{a} f_{2}\left(t-\frac{l}{a}\right)\right\}
$$

Considering Eq. (11a), and recalling $a=\sqrt{H / m}$, Eq. (12) may be rewritten as

$$
\frac{\partial w}{\partial x}(0, t)=-\frac{f_{1}(t)}{H}
$$

Similarly, for $x=l$, we have

$$
\frac{\partial w}{\partial x}(l, t)=\frac{f_{2}(t)}{H}
$$

Equations (13) and (14) show that the dynamic rotational displacements at the cable ends are equal to the ratios between dynamic vertical support forces and the static horizontal tension. 


\subsection{Force method dynamic equation (FMDE)}

When oscillating with small amplitude, the displacement compatibility equation of the cable is [4]

$$
h=-\frac{E A}{l_{e}} \int_{0}^{l} \frac{d^{2} z}{d x^{2}} w d x=\frac{E A}{l_{e}} \frac{m g}{H} \int_{0}^{l} w d x
$$

where $l_{e}, l, E$, and $A$ are respectively curve length, chord length, elastic modulus and cross sectional area of the cable.

Integrating Eq. (1) with respect to $x$ in domain $(0, l)$, and considering Eqs (8), (13), (14) and (15), leads to the governing equation of the system expressed in terms of the dynamic tension increment,

$$
\frac{d^{2} h}{d t^{2}}+\eta^{2} h=\frac{\eta^{2}}{r_{1}}\left[f_{1}+f_{2}+\int_{0}^{l} q(x, t) d x\right]
$$

where

$$
\eta^{2}=\frac{l m g^{2} E A}{l_{e} H^{2}}, r_{1}=\frac{m g l}{H},
$$

and $f_{1}(t), f_{2}(t)$ may be obtained through Eq. (11).

Equation (16a) is the force method dynamic equation (FMDE) for oscillating cables. The explicit solution of Eq. (16a) with initial displacement and velocity of zero may be expressed as

$$
h(t)=\frac{1}{\eta} \int_{0}^{t} \tilde{F}(\tau) \sin (\eta t-\eta \tau) d \tau
$$

where

$$
\tilde{F}(t)=\frac{\eta^{2}}{r_{1}}\left[f_{1}+f_{2}+\int_{0}^{l} q(x, t) d x\right] .
$$

Equation (16b) is known as the Duhamel integral equation and may be evaluated numerically using the procedure described in [3].

\subsection{Free vibration equation}

Substituting Eq. (11) into Eq. (16a) and assuming $q(x, t)=0$, we get

$$
\frac{d^{2} h}{d t^{2}}+\eta^{2} h=\frac{\eta^{2}}{r_{1}}\left\{\frac{2 r_{1}}{r_{2}} \int_{t-l / a}^{t} h(\tau) d \tau-f_{1}\left(t-\frac{l}{a}\right)-f_{2}\left(t-\frac{l}{a}\right)\right\}
$$

where

$$
r_{2}=\frac{l}{a}
$$

Differentiating Eq. (17) with respect to $t$, we get

$$
\frac{d^{3} h}{d t^{3}}+\eta^{2} \frac{d h}{d t}-\frac{2 \eta^{2}}{r_{2}} h=\frac{\eta^{2}}{r_{1}}\left[\frac{-2 r_{1}}{r_{2}} h\left(t-\frac{l}{a}\right)-\frac{d f_{1}\left(t-\frac{l}{a}\right)}{d t}-\frac{d f_{2}\left(t-\frac{l}{a}\right)}{d t}\right]
$$

Substituting $h(t)=\tilde{h} e^{i \omega t}, f_{1}(t)=\tilde{f}_{1} e^{i \omega t}, f_{2}(t)=\tilde{f}_{2} e^{i \omega t}$, into Eqs (11) and (18), we obtain the nonlinear equation defining the natural cable frequencies, $\omega$

$$
\frac{\bar{\omega}}{2}-\frac{\bar{\omega}^{3}}{2 \lambda^{2}}=\tan \frac{\bar{\omega}}{2}
$$

where $\bar{\omega}=\omega l / a, \lambda^{2}=\eta^{2}(l / a)^{2}$. Equation (19) is the same as the frequency equation derived by means of the displacement method [4]. 
(a)

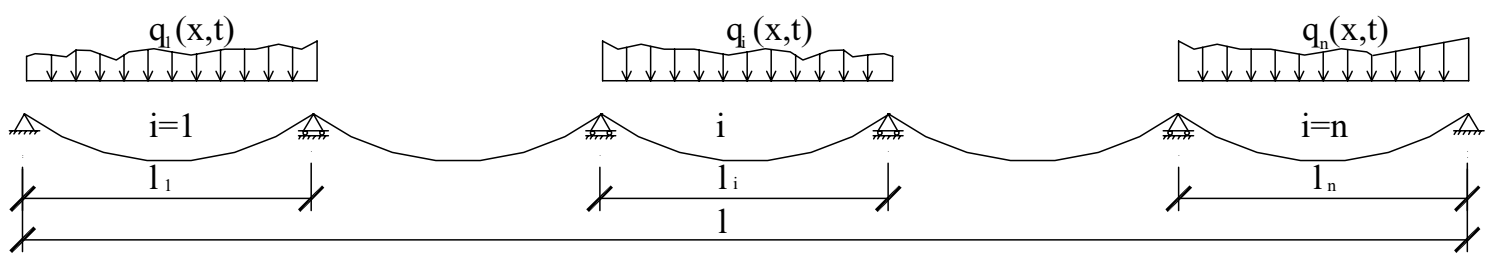

(b)
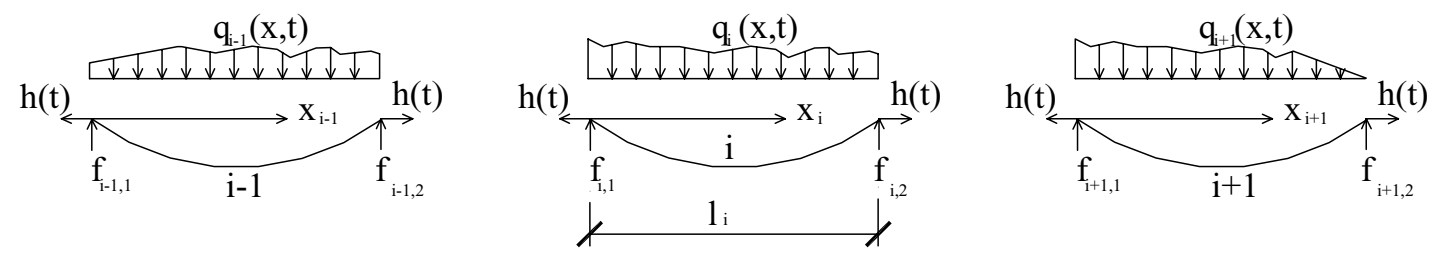

(c)

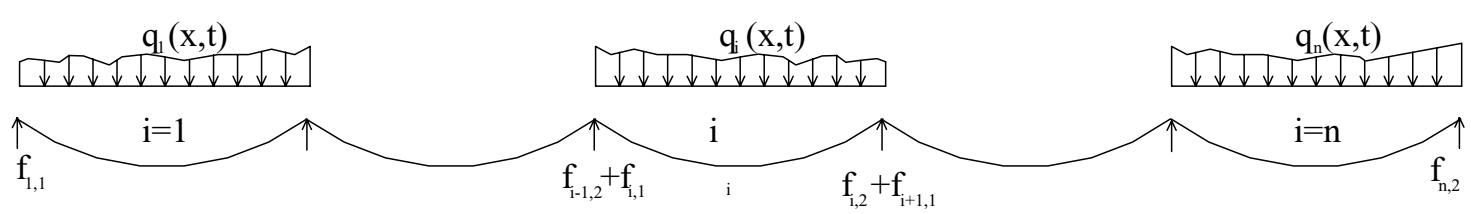

(a) Mechanical model; (b) free-body diagram of the $i^{\text {th }}$ cable segment; (c) forces on the cable system

Fig. 2. Definition diagram for a multi-span cable system.

\section{Force method model for a multi-span cable}

Consider a multi-span cable system (Fig. 2a), which is anchored on rigid supports at each end and supported on rollers at intermediate points [4]. Definitions of the main parameters are as follows: $n$ is the span number; $l_{i}$ is the span of the ith cable segment, $l$ is the total horizontal length of the cable system.

Similar to Eqs (3) and (11), the dynamic displacement and reaction forces for the ith cable segment with two vertically unmovable ends (Fig. 2b) may be developed as

$$
w_{i}\left(x_{i}, t\right)=\frac{1}{2 a} \int_{0}^{t} \int_{x_{i}-a(t-\tau)}^{x_{i}+a(t-\tau)} F_{i}(\xi, \tau) d \xi d \tau
$$

with

$$
\begin{aligned}
& F_{i}\left(x_{i}, t\right)=\left\{\begin{array}{cl}
\frac{h}{m} \frac{d^{2} z_{i}}{d x_{i}^{2}}+\frac{f_{i, 1} \delta\left(x_{i}\right)+f_{i, 2} \delta\left(x_{i}-l_{i}\right)+q_{i}}{m} & 0 \leqslant x_{i} \leqslant l_{i} \text { and } t \geqslant 0 \\
0 & \text { others }
\end{array}\right. \\
& f_{i, 1}(t)=\left\{\begin{array}{cr}
0 & t<0 \\
-f_{i, 2}\left(t-\frac{l_{i}}{a}\right)+a \int_{t-\frac{l_{i}}{a}}^{t}\left\{\frac{m g}{H} h(\tau)-q_{i}[a(t-\tau), \tau]\right\} d \tau & t \geqslant 0
\end{array}\right.
\end{aligned}
$$

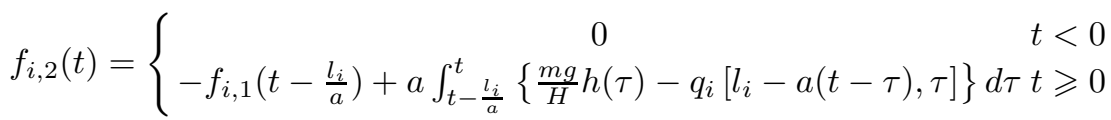

where $x_{i}$ is the axial coordinate; $m$ is the mass density; $H$ and $h(t)$ are respectively the horizontal components of static tension and the increment of dynamic tension; $f_{i, 1}(t), f_{i, 2}(t), q_{i}\left(x_{i}, t\right)$ are the reaction forces and the external load; $w_{i}\left(x_{i}, t\right), z_{i}\left(x_{i}\right)$ are respectively the dynamic displacement response and the static curve of the cable under equilibrium load $\left(q_{i}=0\right)$. 
Considering all the forces acting on the whole cable system (Fig. 2c), the dynamic force method equation of (16a) may be rewritten as

$$
\frac{d^{2} h}{d t^{2}}+\eta^{2} h=\sum_{i=1}^{n} \tilde{F}_{i}(t)
$$

with the explicit solution expressed as

$$
h(t)=\frac{1}{\eta} \int_{0}^{t} \sum_{i=1}^{n} \tilde{F}_{i}(\tau) \sin (\eta t-\eta \tau) d \tau
$$

where

$$
\tilde{F}_{i}(t)=\frac{\eta^{2}}{r_{1}}\left[f_{i, 1}+f_{i, 2}+\int_{0}^{l_{i}} q_{i}(x, t) d x\right], \eta^{2}=\frac{l m g^{2} E A}{l_{e} H^{2}}, r_{1}=\frac{m g l}{H}, l_{e}
$$

is the curve length of the whole cable, other parameters have the same meanings as the single cable system.

For free-vibration analysis, assuming $q_{i}=0$ and substituting

$$
h(t)=\tilde{h} e^{i \omega t}, f_{i, 1}(t)=\tilde{f}_{i, 1} e^{i \omega t}, f_{i, 2}(t)=\tilde{f}_{i, 2} e^{i \omega t}
$$

into Eqs (20c), (20d) and (21a), we obtain the nonlinear equation defining the natural cable frequencies, $\omega$

$$
\frac{\bar{\omega}}{2}-\frac{\bar{\omega}^{3}}{2 \lambda^{2}}=\sum_{i=1}^{n} \tan \left(\frac{\alpha_{i} \bar{\omega}}{2}\right)
$$

where

$$
\bar{\omega}=\omega l / a, \lambda^{2}=\eta^{2}(l / a)^{2}, \alpha_{i}=l_{i} / l .
$$

Equation (22) is the same as the frequency equation derived by means of the displacement method [4].

\section{Nonlinear model}

\subsection{Single-span cable model}

Considering damping forces and the nonlinear effects on the dynamic response, the motion Eq. (1) may be rewritten as

$$
(H+\hat{h}) \frac{\partial^{2} \hat{w}}{\partial x^{2}}+\hat{h} \frac{d^{2} z}{d x^{2}}+q=m \frac{\partial^{2} \hat{w}}{\partial t^{2}}+c \frac{\partial \hat{w}}{\partial t}
$$

where $\hat{h}$ and $\hat{w}$ are respectively the horizontal component of the additional dynamic tension and the dynamic displacement response, where the values of both take account of nonlinear effects; $c$ is the damping coefficient.

The displacement compatibility Eq. (15) may be rewritten as

$$
\hat{h}=\frac{E A}{l_{e}}\left[\frac{m g}{H} \int_{0}^{l} w d x+\frac{1}{2} \int_{0}^{l}\left(\frac{\partial w}{\partial x}\right)^{2} d x\right]
$$

Assuming

$$
\begin{aligned}
& \hat{h}=\sum_{k=1}^{N} \varepsilon^{k} \hat{h}_{k} \\
& \hat{w}=\sum_{k=1}^{N} \varepsilon^{k} \hat{w}_{k}
\end{aligned}
$$




$$
\begin{gathered}
q=\varepsilon \hat{q}_{1} \\
c=\varepsilon \hat{c}
\end{gathered}
$$

where $\varepsilon$ is an arbitrarily small parameter; and substituting Eq. (25) into Eqs (23) and (24), considering $N=3$ and equating all terms of like order, $\varepsilon, \varepsilon^{2}$, and $\varepsilon^{3}$, produces

$$
\begin{aligned}
& \varepsilon: \quad H \frac{\partial^{2} \hat{w}_{1}}{\partial x^{2}}+\hat{h}_{1} \frac{d^{2} z}{d x^{2}}+\hat{q}_{1}=m \frac{\partial^{2} \hat{w}_{1}}{\partial t^{2}} \\
& \hat{h}_{1}=\frac{E A}{l_{e}} \frac{m g}{H} \int_{0}^{l} \hat{w}_{1} d x \\
& \varepsilon^{2}: \quad H \frac{\partial^{2} \hat{w}_{2}}{\partial x^{2}}+\hat{h}_{2} \frac{d^{2} z}{d x^{2}}+\hat{h}_{1} \frac{\partial^{2} \hat{w}_{1}}{\partial x^{2}}-\hat{c} \frac{\partial \hat{w}_{1}}{\partial t}=m \frac{\partial^{2} \hat{w}_{2}}{\partial t^{2}} \\
& \hat{h}_{2}=\frac{E A}{l_{e}}\left[\frac{m g}{H} \int_{0}^{l} \hat{w}_{2} d x+\frac{1}{2} \int_{0}^{l}\left(\frac{\partial \hat{w}_{1}}{\partial x}\right)^{2} d x\right] \\
& \varepsilon^{3}: \quad H \frac{\partial^{2} \hat{w}_{3}}{\partial x^{2}}+\hat{h}_{3} \frac{d^{2} z}{d x^{2}}+\hat{h}_{1} \frac{\partial^{2} \hat{w}_{2}}{\partial x^{2}}+\hat{h}_{2} \frac{\partial^{2} \hat{w}_{1}}{\partial x^{2}}-\hat{c} \frac{\partial \hat{w}_{2}}{\partial t}=m \frac{\partial^{2} \hat{w}_{3}}{\partial t^{2}} \\
& \hat{h}_{3}=\frac{E A}{l_{e}}\left[\frac{m g}{H} \int_{0}^{l} \hat{w}_{3} d x+\int_{0}^{l}\left(\frac{\partial \hat{w}_{1}}{\partial x}\right)\left(\frac{\partial \hat{w}_{2}}{\partial x}\right) d x\right]
\end{aligned}
$$

where Eq. (26) is the same as the linearised model of Eqs (1) and (15) with

$$
\varepsilon \hat{h}_{1}=h \text { and } \varepsilon \hat{w}_{1}=w
$$

Assuming

$$
\hat{h}_{2}=\hat{h}_{2 a}+\frac{E A}{2 l_{e}} \int_{0}^{l}\left(\frac{\partial \hat{w}_{1}}{\partial x}\right)^{2} d x
$$

and

$$
\hat{h}_{3}=\hat{h}_{3 a}+\frac{E A}{l_{e}} \int_{0}^{l}\left(\frac{\partial \hat{w}_{1}}{\partial x}\right)\left(\frac{\partial \hat{w}_{2}}{\partial x}\right) d x
$$

Equations (27) and (28) may be rewritten as

$$
\begin{aligned}
& H \frac{\partial^{2} \hat{w}_{k}}{\partial x^{2}}+\hat{h}_{k a} \frac{d^{2} z}{d x^{2}}+\hat{q}_{k}=m \frac{\partial^{2} \hat{w}_{k}}{\partial t^{2}} k=2,3 \\
& \hat{h}_{k a}=\frac{E A}{l_{e}} \frac{m g}{H} \int_{0}^{l} \hat{w}_{k} d x \quad k=2,3 \\
& \hat{q}_{2}=\hat{h}_{1} \frac{\partial^{2} \hat{w}_{1}}{\partial x^{2}}-\frac{m g}{H} \frac{E A}{2 l_{e}} \int_{0}^{l}\left(\frac{\partial \hat{w}_{1}}{\partial x}\right)^{2} d x-\hat{c} \frac{\partial \hat{w}_{1}}{\partial t}
\end{aligned}
$$

and

$$
\hat{q}_{3}=\hat{h}_{1} \frac{\partial^{2} \hat{w}_{2}}{\partial x^{2}}+\hat{h}_{2} \frac{\partial^{2} \hat{w}_{1}}{\partial x^{2}}-\frac{m g}{H} \frac{E A}{l_{e}} \int_{0}^{l}\left(\frac{\partial \hat{w}_{1}}{\partial x}\right)\left(\frac{\partial \hat{w}_{2}}{\partial x}\right) d x-\hat{c} \frac{\partial \hat{w}_{2}}{\partial t}
$$


where Eqs (30a) and (30b) have similar form to Eqs (1) and (15). The force method governing equation and explicit solution for $\hat{h}_{k a}$ are

$$
\begin{aligned}
& \frac{d^{2} \hat{h}_{k a}}{d t^{2}}+\eta^{2} \hat{h}_{k a}=\hat{F}_{k}(t) k=2,3 \\
& \hat{h}_{k a}(t)=\frac{1}{\eta} \int_{0}^{t} \hat{F}_{k}(\tau) \sin (\eta t-\eta \tau) d \tau
\end{aligned}
$$

with

$$
\begin{aligned}
& \hat{F}_{k}(t)=\frac{\eta^{2}}{r_{1}}\left[\hat{f}_{1, k}+\hat{f}_{2, k}+\int_{0}^{l} \hat{q}_{k}(x, t) d x\right] \\
& \hat{f}_{1, k}(t)=\left\{\begin{array}{cc}
0 & t<0 \\
-\hat{f}_{2, k}\left(t-\frac{l}{a}\right)+a \int_{t-\frac{l}{a}}^{t}\left\{\frac{m g}{H} \hat{h}_{k a}(\tau)-\hat{q}_{k}[a(t-\tau), \tau]\right\} d \tau & t \geqslant 0
\end{array} k=2,3\right. \\
& \hat{f}_{2, k}(t)=\left\{\begin{array}{cc}
0 & t<0 \\
-\hat{f}_{1, k}\left(t-\frac{l}{a}\right)+a \int_{t-\frac{l}{a}}^{t}\left\{\frac{m g}{H} \hat{h}_{k a}(\tau)-\hat{q}_{k}[l-a(t-\tau), \tau]\right\} d \tau & t \geqslant 0
\end{array} k=2,3\right.
\end{aligned}
$$

\subsection{Multi-span cable model}

Similar to the single-span cable model, assuming the nonlinear tension response has the same form as Eq. (25a), the external loading and nonlinear displacement response of the ith cable segment may be expressed as

$$
\begin{aligned}
& q_{i}=\varepsilon \hat{q}_{i, 1} \\
& \hat{w}_{i}=\sum_{k=1}^{N} \varepsilon^{k} \hat{w}_{i, k}
\end{aligned}
$$

where the linearised displacement response $w_{i, 1}$ for the ith cable segment may be calculated based on Eqs (20a) and (20b).

Thus loads due to nonlinear responses may be expressed as

$$
\hat{q}_{i, 2}=\hat{h}_{1} \frac{\partial^{2} \hat{w}_{i, 1}}{\partial x_{i}^{2}}-\frac{m g}{H} \frac{E A}{2 l_{e}} \int_{0}^{l_{i}}\left(\frac{\partial \hat{w}_{i, 1}}{\partial x}\right)^{2} d x-\hat{c} \frac{\partial \hat{w}_{i, 1}}{\partial t}
$$

and

$$
\hat{q}_{i, 3}=\hat{h}_{1} \frac{\partial^{2} \hat{w}_{i, 2}}{\partial x_{i}^{2}}+\hat{h}_{2} \frac{\partial^{2} \hat{w}_{i, 1}}{\partial x_{i}^{2}}-\frac{m g}{H} \frac{E A}{l_{e}} \int_{0}^{l_{i}}\left(\frac{\partial \hat{w}_{i, 1}}{\partial x}\right)\left(\frac{\partial \hat{w}_{i, 2}}{\partial x}\right) d x-\hat{c} \frac{\partial \hat{w}_{i, 2}}{\partial t}
$$

The nonlinear response $h_{k a}$ may be calculated based on the following equation

$$
\frac{d^{2} \hat{h}_{k a}}{d t^{2}}+\eta^{2} \hat{h}_{k a}=\sum_{i=1}^{n} \hat{F}_{i, k}(t) k=2,3
$$

with the explicit solution of

$$
\hat{h}_{k a}(t)=\frac{1}{\eta} \int_{0}^{t} \sum_{i=1}^{n} \hat{F}_{i, k}(\tau) \sin (\eta t-\eta \tau) d \tau
$$

where 
Table 1

Natural frequencies for a single span cable

\begin{tabular}{lcccccccc}
\hline No. & 1 & 2 & 3 & 4 & 5 & 6 & 7 & 8 \\
\hline$f_{z}\left(\mathbf{H}_{z}\right)$ & 0.149 & 0.256 & 0.356 & 0.416 & 0.479 & 0.579 & 0.682 & 0.787 \\
\hline
\end{tabular}

$-F M \quad \times \quad D M(n=8) \cdots \cdots \cdot D(n=4)$

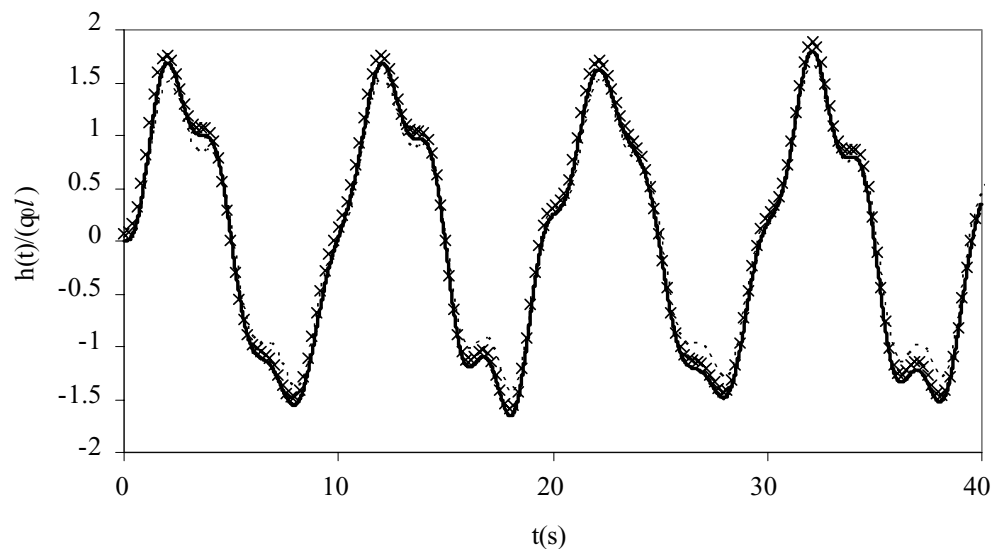

Fig. 3. Linearised dynamic response $h(t)$ of a single-span cable $\left(f=0.1 \mathrm{H}_{z}\right)$.

$$
\begin{aligned}
& \hat{F}_{i, k}(t)=\frac{\eta^{2}}{r_{1}}\left[\hat{f}_{i, 1, k}+\hat{f}_{i, 2, k}+\int_{0}^{l_{i}} \hat{q}_{i, k}(x, t) d x\right] \\
& \hat{f}_{i, 1, k}(t)=\left\{\begin{array}{cr}
0 & t<0 \\
-\hat{f}_{i, 2, k}\left(t-\frac{l_{i}}{a}\right)+a \int_{t-\frac{l_{i}}{a}}^{t}\left\{\frac{m g}{H} \hat{h}_{k a}(\tau)-\hat{q}_{i, k}[a(t-\tau), \tau]\right\} d \tau & t \geqslant 0
\end{array}\right. \\
& \hat{f}_{i, 2, k}(t)=\left\{\begin{array}{cr}
0 & t<0 \\
-\hat{f}_{i, 1, k}\left(t-\frac{l_{i}}{a}\right)+a \int_{t-\frac{l_{i}}{a}}^{t}\left\{\frac{m g}{H} \hat{h}_{k a}(\tau)-\hat{q}_{i, k}\left[l_{i}-a(t-\tau), \tau\right]\right\} d \tau t \geqslant 0
\end{array}\right.
\end{aligned}
$$

\section{Examples}

Example 1 Consider a single-span cable with the following parameters [8]: $l=1369.36 \mathrm{~m}$, sag $d=123.52 \mathrm{~m}$, $m=5951.04 \mathrm{~kg} / \mathrm{m}, E=2.0 \times 10^{11} \mathrm{~N} / \mathrm{m}^{2}, A=0.759 \mathrm{~m}^{2}, H=122600 \mathrm{kN}$. Based on Eq. (19), the first eight natural frequencies for in-plane symmetric modes may be obtained as shown in Table 1. The first three frequencies with the same values were also given in [8].

To verify the force methods presented in the paper, we consider a uniformly distributed excitation $q(x, t)=$ $q_{0} \sin 2 \pi f t, f=0.1 \mathrm{H}_{z}$. For small amplitude excitation the linearised model is applicable, and the undamped responses from the force method (FM) are compared with those from the mode superposition method using just the first 4 modes (DM, $n=4$ ) and also when using the first 8 modes (DM, $n=8$ ). The comparison of results in Fig. 3 shows good agreement between the FM and DM $(n=8)$ models. The comparison also shows that a traditional displacement method, DM $(n=4)$ including only a few linear mode functions (usually 2 to 4 ) may result in inaccurate predictions for long cables.

The nonlinear responses of the structure subjected to excitations with different amplitudes and frequencies are compared with ABAQUS results in Figs 4 and 5. In the ABAQUS model, the structure was divided into 1000 truss elements and a dynamic-implicit analysis procedure was implemented. 
Table 2

Table 2 Natural frequencies $f_{z}\left(\mathbf{H}_{z}\right)$ for a three-span cable system

\begin{tabular}{cccl}
\hline No. & ABAQUS & Equation (22) & Mode type \\
\hline 1 & 0.228 & 0.228 & symmetric \\
2 & 0.302 & - & asymmetric \\
3 & 0.306 & - & asymmetric \\
4 & 0.437 & 0.438 & symmetric \\
5 & 0.529 & 0.538 & symmetric \\
6 & 0.612 & - & asymmetric \\
\hline
\end{tabular}

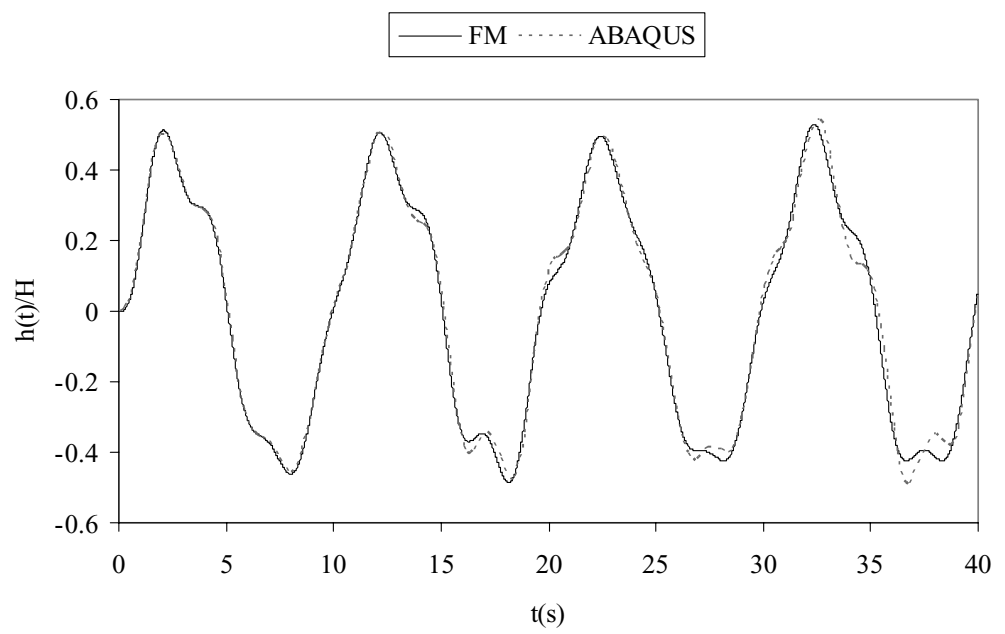

Fig. 4. Nonlinear dynamic response $h(t)$ of a single-span cable $\left(f=0.1 \mathrm{H}_{z}, q_{0}=0.3 H / l\right)$.
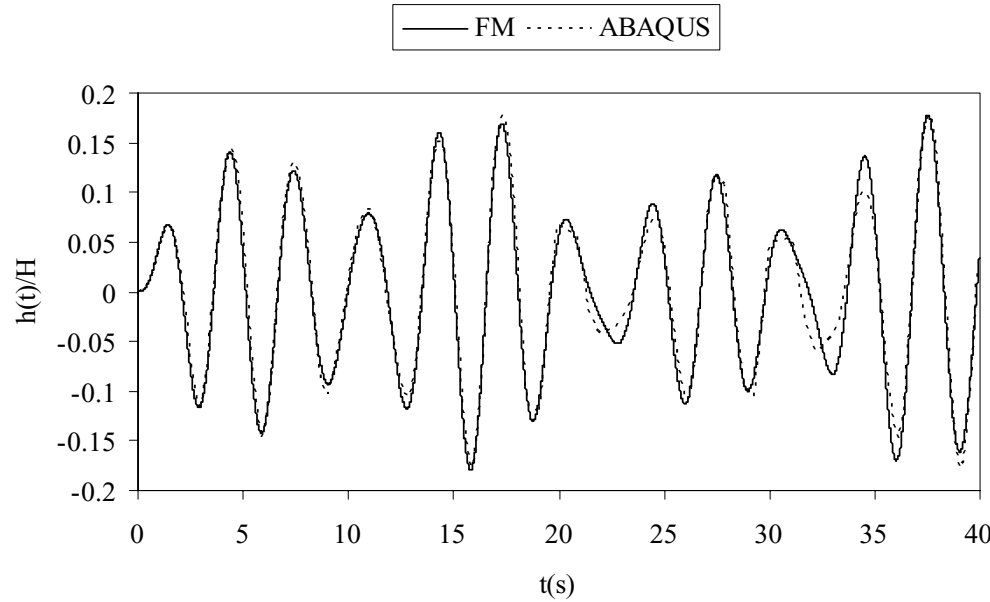

Fig. 5. Nonlinear dynamic response $h(t)$ of a single-span cable $\left(f=0.3 \mathrm{H}_{z}, q_{0}=0.03 \mathrm{H} / l\right)$.

Example 2 Consider a three-span cable system with the following parameters [2]: $l=800 \mathrm{~m}, l_{1}=200 \mathrm{~m}, l_{2}=$ $400 \mathrm{~m}, l_{3}=200 \mathrm{~m}, m=2.3 \mathrm{~kg} / \mathrm{m}, E A=53,000,000 \mathrm{~N}, H=34,500 \mathrm{~N}, c=0.04 \mathrm{~N}-\mathrm{s} / \mathrm{m}^{2}$. The first six natural frequencies for in-plane modes are shown in Table 2 with the corresponding vibration modes shown in Fig. 6.

To verify the force method model presented, two uniformly distributed excitations with different amplitudes and frequencies are applied on the structure, the nonlinear response is compared with ABAQUS results in Figs 7 and 8. In the ABAQUS model, the structure was divided into 1000 truss elements and a dynamic-implicit analysis procedure was implemented. 


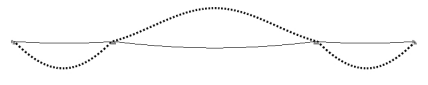

Mode $1\left(f_{z}=0.228 \mathrm{~Hz}\right)$

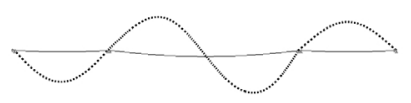

Mode $3\left(\mathrm{f}_{\mathrm{z}}=0.306 \mathrm{~Hz}\right)$

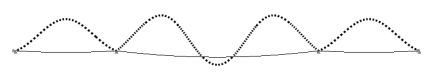

Mode $5\left(\mathrm{f}_{\mathrm{z}}=0.529 \mathrm{~Hz}\right)$

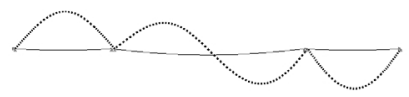

Mode $2\left(f_{z}=0.302 \mathrm{~Hz}\right)$

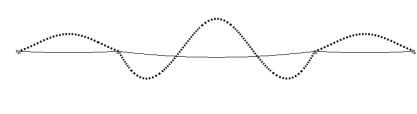

Mode $4\left(\mathrm{f}_{\mathrm{z}}=0.437 \mathrm{~Hz}\right)$

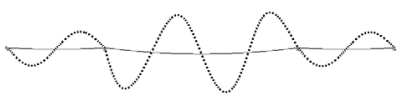

Mode $6\left(\mathrm{f}_{\mathrm{z}}=0.612 \mathrm{~Hz}\right)$

Fig. 6. Vibration modes of a three-span cable based on ABAQUS.
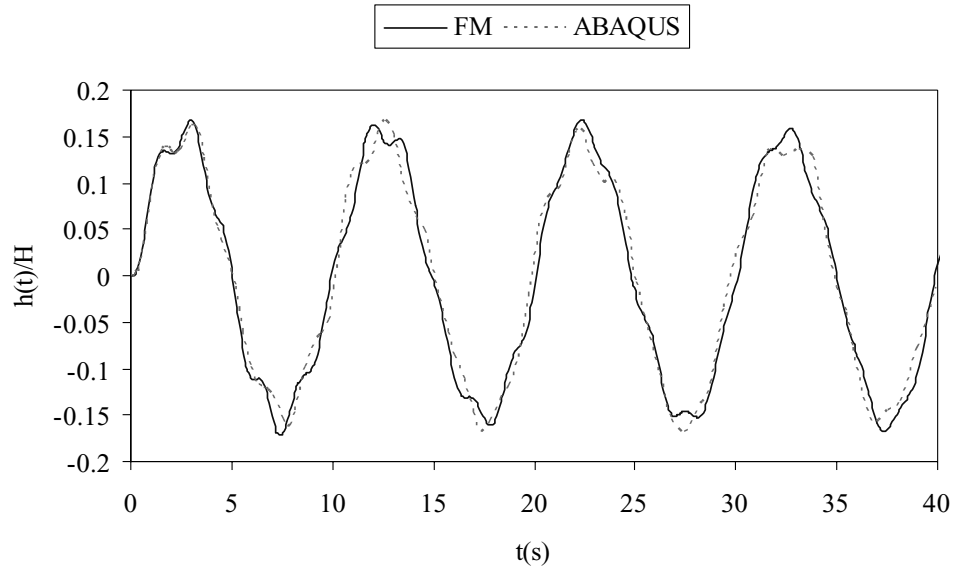

Fig. 7. Dynamic response $h(t)$ of a three-span cable $\left(f=0.1 \mathrm{H}_{z}, q_{0}=0.1 H / l\right)$.

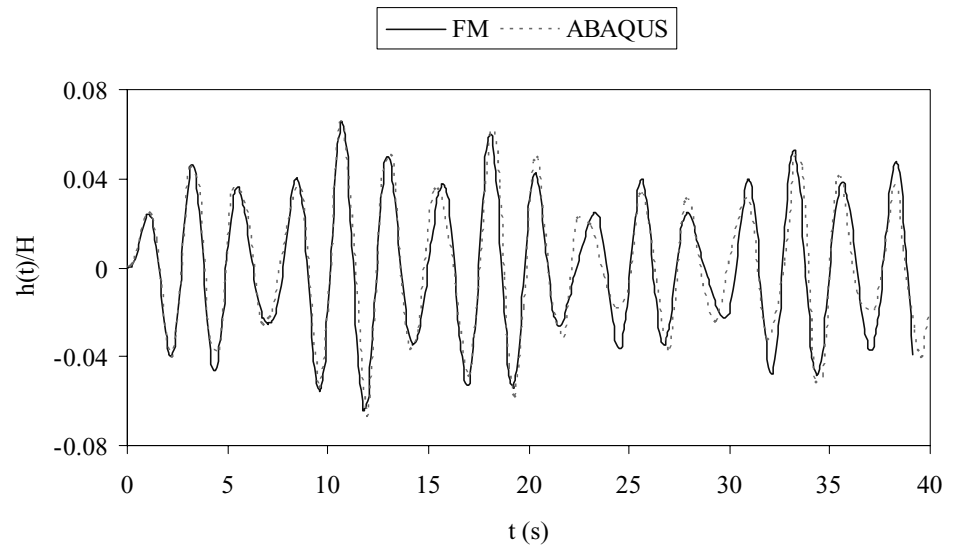

Fig. 8. Dynamic response $h(t)$ of a three-span cable $\left(f=0.4 \mathrm{H}_{z}, q_{0}=0.01 H / l\right)$. 


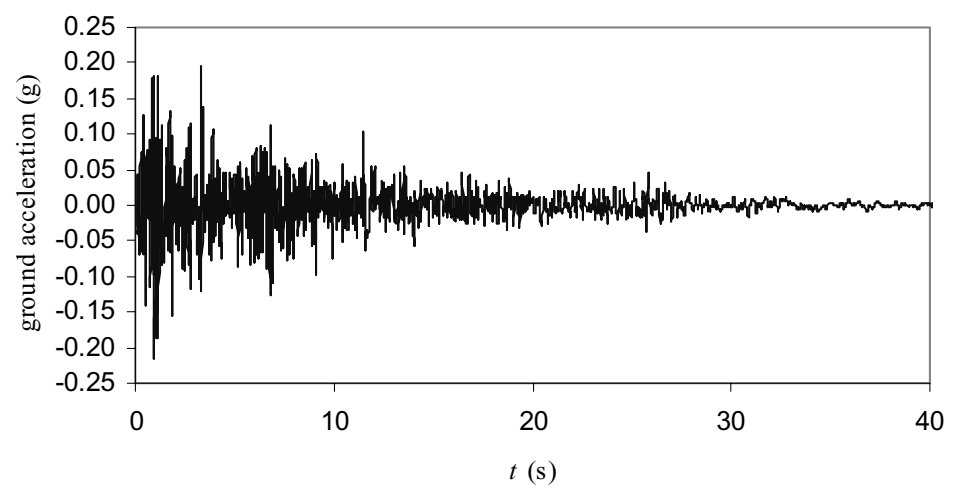

Fig. 9. Accelerogram from $1940 \mathrm{El}$ Centro earthquake (vertical component).

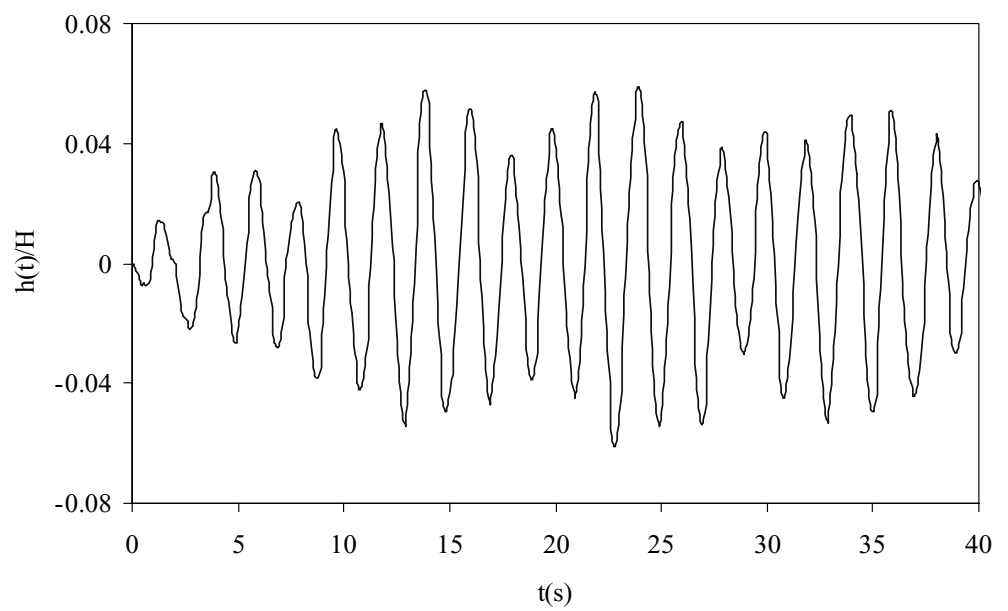

Fig. 10. Dynamic response $h(t)$ of a three-span cable subject to vertical earthquake excitation.

To illustrate a practical engineering application a vertical ground motion excitation based on the vertical component of the 1940 El Centro earthquake record (Fig. 9) was applied, and the nonlinear force method model used to calculate the cable responses shown in Fig. 10.

\section{Conclusions}

An analysis method, named the force method dynamic equation (FMDE), for the oscillation of shallow, single or multi-span cables, has been developed in the form of a single-degree-of-freedom (SDOF) system in terms of the additional dynamic tension. The natural frequencies derived from the FMDE are shown to be identical to those obtained from the conventional displacement method (DM).

Nonlinear governing equations are developed by considering the quadratic and cubic terms of the dynamic displacements. Numerical results show that the FM model provides dynamic response predictions that are significantly more accurate than those obtained by a traditional DM mode superposition with 2 to 4 linear modes included. Accuracy is comparable with a DM mode superposition analysis using an appropriately larger number of linear modes ( 8 in the example considered). Comparison with ABAQUS simulations confirms the good accuracy of the FM model. 


\section{References}

[1] F. Benedettini, G. Rega and R. Alaggio, Non-linear oscillations of a four-degree-of-freedom model of a suspended cable under multiple internal response conditions, Journal of Sound and Vibration 182(5) (1995), 775-798.

[2] X.M. Chen, Galloping Control for Transmission Lines, Ph.D. Dissertation, Tongji University, Shanghai, China, 2002.

[3] R. Clough and J. Penzien, Dynamics of Structures, Computers and Structures, Inc., Berkeley, California, USA, 2003.

[4] H.M. Irvine, Cable Structures, The MIT Press, Cambridge, New York, 1981

[5] F. John, Partial Differential Equations, Springer-Verlag, New York, 1982.

[6] J.W. Larsen and S.R.K. Nielsen, Nonlinear stochastic response of a shallow cable, International Journal of Non-linear Mechanics 41(5) (2006), 629-643.

[7] J.W. Leonard, Tension Structures, McGraw-Hill Book Company, New York, 1988.

[8] Y.Q. Ni, W.J. Lou and J.M. Ko, A hybrid pseudo force/Laplace transform method for non-linear transient response of a suspended cable, Journal of Sound and Vibration 238(2) (2000), 189-214.

[9] G. Rega, F. Vestroni and F. Benedettini, Parametric analysis of large amplitude free vibrations of a suspended cable, International Journal of Solids and Structures 20(2) (1984), 95-105.

[10] P. Warnitchai, Y. Fujino and T. Susumpow, A non-linear dynamic model for cables and its application to a cable-structures system, Journal of Sound and Vibration 187(4) (1995), 695-712.

[11] Z.G Ying, Y.Q. Ni and J.M. Ko, Parametrically excited instability analysis of a semi-actively controlled cable, Engineering Structures 29(4) (2007), 567-575.

[12] Z. You, Sensitivity analysis based on the force method for deployable cable-stiffened structures, Engineering Optimization 29(1-4) (1997), 429-441.

[13] Q. Zhou, S.R.K. Nielsen and W.L. Qu, Semi-active control of three dimensional vibrations of an inclined sag cable with magnetorheological dampers, Journal of Sound and Vibration 296(1) (2006), 1-22. 

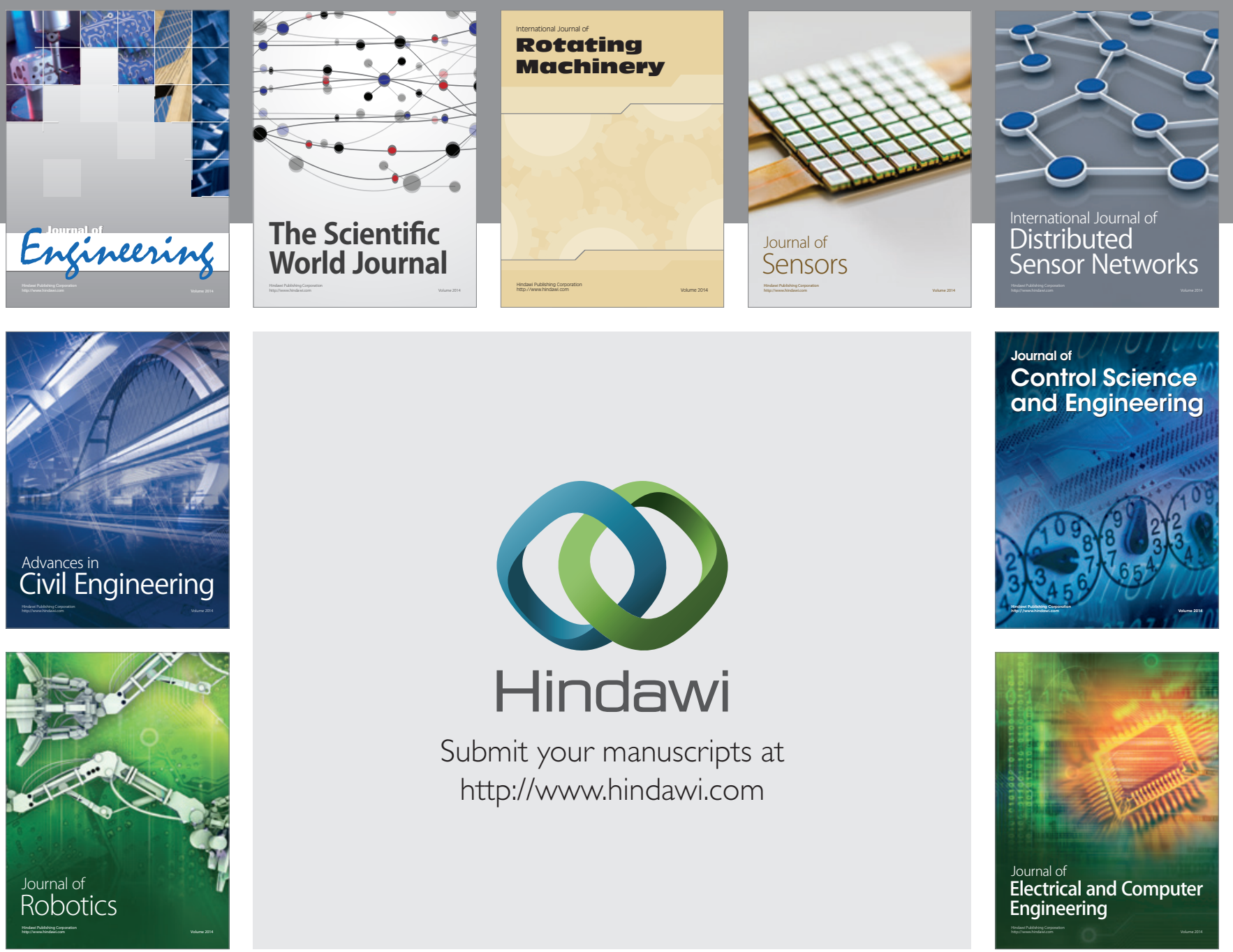

Submit your manuscripts at

http://www.hindawi.com
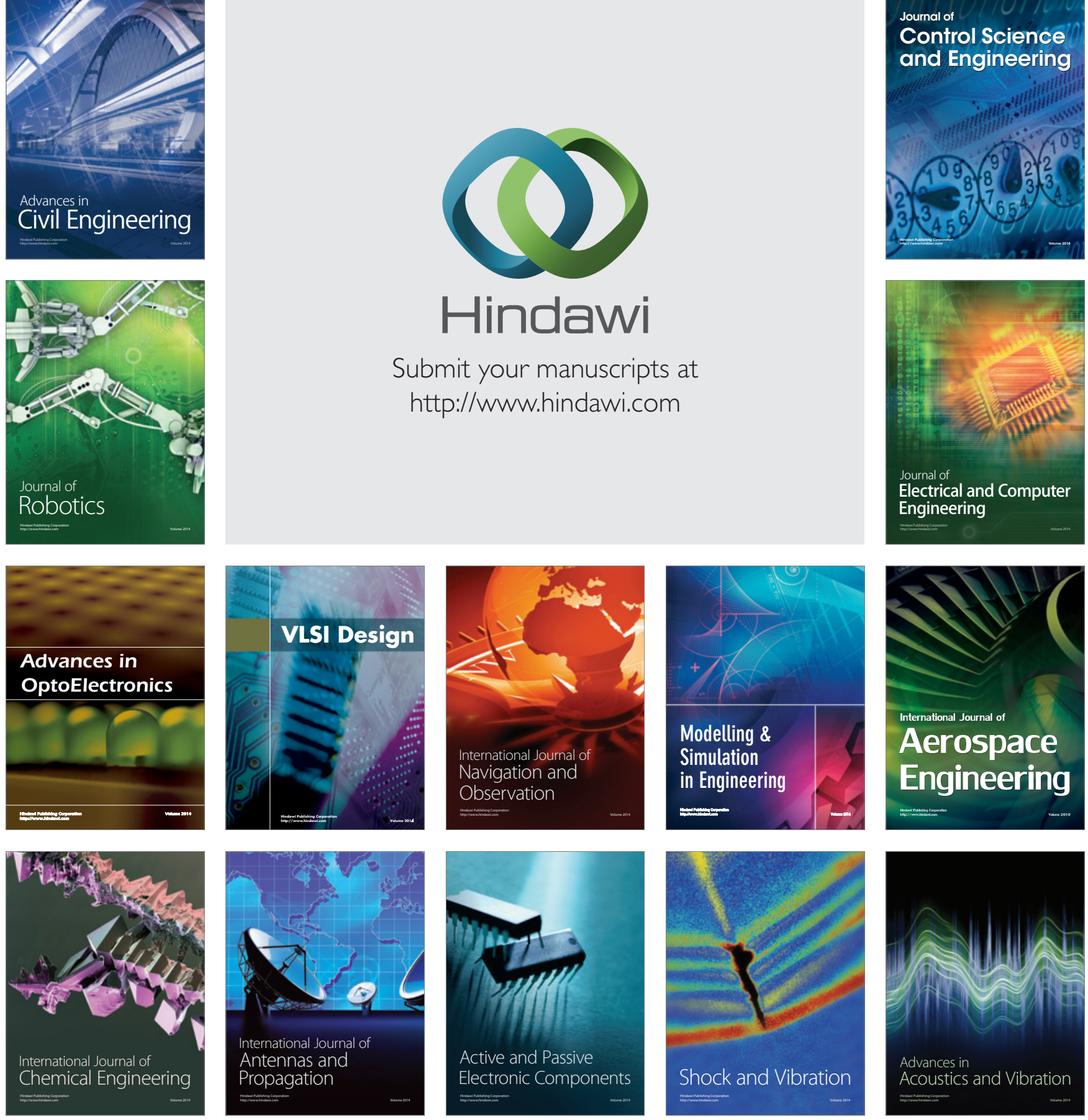